\title{
Rare decays at CMS
}

\author{
Jónatan Piedra*广 \\ IFCA (CSIC - Universidad de Cantabria) \\ E-mail: piedra@cern.ch
}

Searches for flavour changing neutral currents (FCNC) in events with top quarks and the $\mathrm{Z}$ or the Higgs boson are presented by the CMS Collaboration. These searches use proton-proton collision events taken by the CMS experiment at the Large Hadron Collider (LHC), built by the European Organization for Nuclear Research (CERN). Upper limits at 95\% confidence level are set on the branching fractions of $\mathrm{t} \rightarrow \mathrm{qZ}$ and $\mathrm{t} \rightarrow \mathrm{qH}$ decays. In addition, angular distributions of $\mathrm{B}^{0}$ and $\mathrm{B}^{+}$ decays are presented. These measurements are found to be consistent with predictions based on the standard model.

XIV International Conference on Heavy Quarks and Leptons (HQL2018)

May 27 - June 1, 2018

Yamagata Terrsa, Yamagata, Japan

\footnotetext{
${ }^{*}$ Speaker.

${ }^{\dagger}$ On behalf of the CMS Collaboration.
} 


\section{1. $\mathrm{FCNC}$ in $\mathrm{tZq} \rightarrow 3 \ell$}

In the standard model (SM), FCNC are forbidden at tree level and highly suppressed at higher orders. Several extensions of the SM enhance the FCNC branching fractions and can be probed at the LHC; the new couplings can also provide for flavour changing single top quark production in association with a $\mathrm{Z}$ or a Higgs boson. This analysis [1] uses proton-proton collision data accumulated during the 2016 running period, corresponding to an integrated luminosity of $35.9 \mathrm{fb}^{-1}$ at a centre-of-mass energy of $13 \mathrm{TeV}$ collected by the CMS detector [2]. The analysis focuses on the experimental search for evidence of FCNC vertex (referred to as $t Z q$ ) with a top quark, a $Z$ boson, and a quark q that is either up or charm. In the final state we expect one jet originating from a b quark, a $\mathrm{W}$ boson, a $\mathrm{Z}$ boson, and (in the case of FCNC top quark decay) a jet originating from the up or charm quark. As we limit the analysis to electronic and muonic decays of both the $\mathrm{W}$ and the $\mathrm{Z}$ boson, events are selected requiring exactly 3 leptons containing one opposite sign, same flavour pair; at least 1 jet and at most 3 jets; and the transverse mass of the $\mathrm{W}$ boson below $300 \mathrm{GeV}$. Four different lepton channels are considered (3e, $2 \mathrm{e} 1 \mu, 1 \mathrm{e} 2 \mu, 3 \mu)$ and two signal regions are constructed using the jet multiplicity, the single top quark (STSR) and the top quark pair (TTSR) FCNC. A simultaneous global fit is performed taking into account both signal regions and background regions for the four lepton channels with the help of boosted decision trees (BDT) used to discriminate between signal and background. The resulting discriminating output variables from each BDT are shown in Figure 1. The signal strength and significance are computed treating all systematic uncertainties as nuisance parameters and are constrained from the fit which uses templates in the different signal and background regions for each of the four different lepton channels. The resulting observed (expected) limits where both couplings are non-vanishing are shown in Figure 2. No significant deviation is observed from the predicted background. Observed (expected) upper limits at 95\% CL are set on the branching fractions of top quark decays: $\mathscr{B}(\mathrm{t} \rightarrow \mathrm{uZ})<0.024 \%(0.015 \%)$ and $\mathscr{B}(\mathrm{t} \rightarrow \mathrm{cZ})<0.045 \%(0.037 \%)$, assuming one non-vanishing coupling at a time.

\section{2. $\mathrm{FCNC}$ in $\mathrm{tH} \rightarrow \mathrm{bb}$}

In this analysis [3] we search for FCNC in events with the top quark and the Higgs boson, but considering only the Higgs boson decays to b quarks. The tH FCNC interaction is studied in two channels: the associated production of a single top quark with the Higgs boson (ST), and the FCNC decays of top quarks in $\bar{t} \bar{t}$ semileptonic events (TT). As before, the data sample corresponds to an integrated luminosity of $35.9 \mathrm{fb}^{-1}$. Events with exactly one isolated lepton (electron or muon) are selected, and at least 3 jets are required to be present. As signal events contain three $b$ quarks, we require that at least two jets are identified as b quark jets by the combined secondary vertex (CSV) b-tagging algorithm. In order to optimize the sensitivity to the signal event selection, events are split into five categories based on the total number of reconstructed jets and on the number of b-tagged jets. Using the energy and momenta of all particles, a full kinematic reconstruction of the event is performed for several signal (ST and TT) and background (tit) hypotheses. The reconstruction is performed for all possible permutations of the b-tagged jets to be associated with the decay products of the Higgs boson or the top quark. The reconstructed kinematic variables for each 

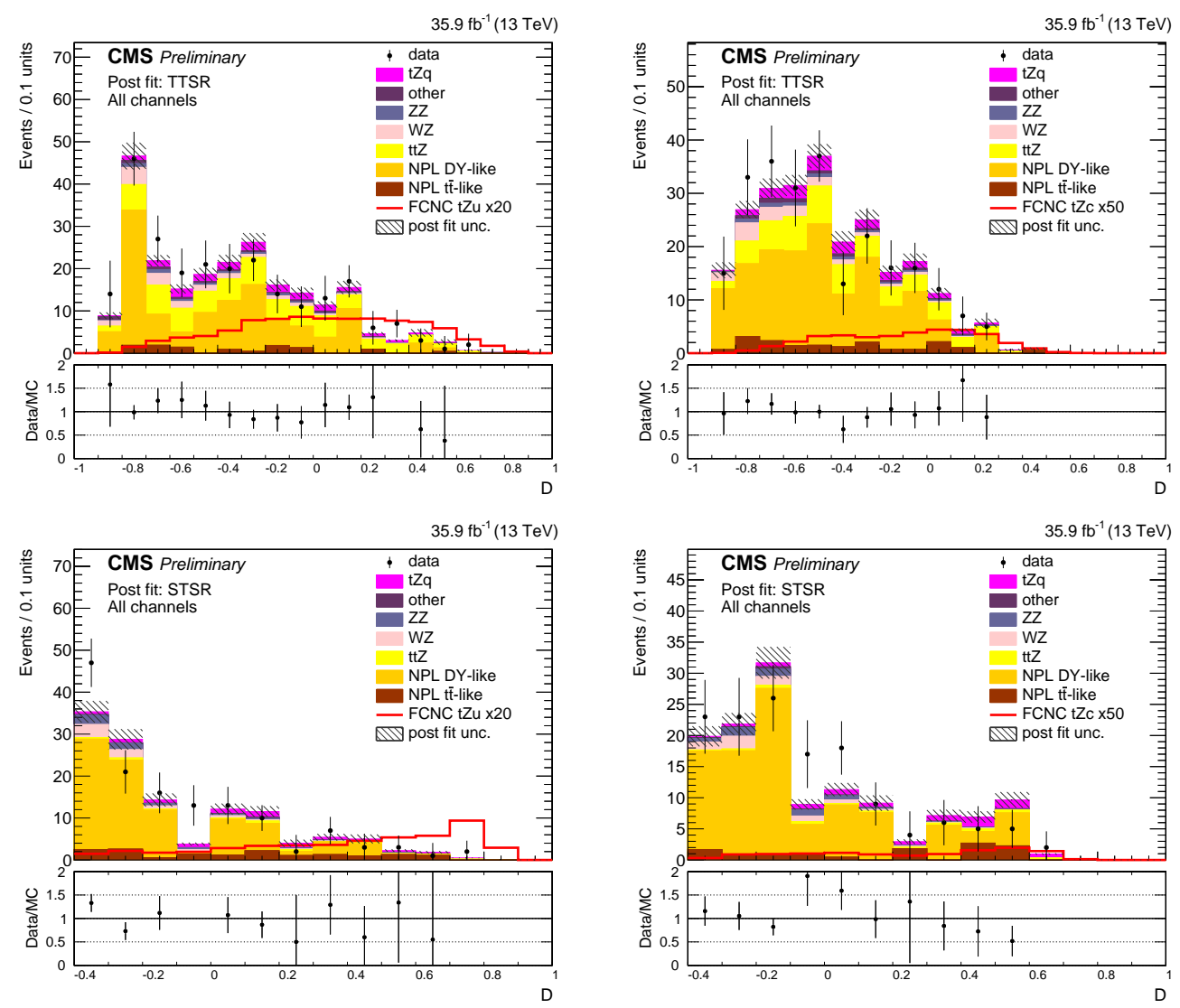

Figure 1: The discriminating variable distribution after the fit for all different leptonic channels [1]. Upper left: top quark pair $\mathrm{tZu}$; upper right: top quark pair tZc; lower left: single top quark $\mathrm{tZu}$; lower right: single top quark tZc.

permutation are inputs for a multivariate analysis that uses a BDT, trained to distinguish the correct from the wrong $b$ jet assignments. In addition, kinematic variables from the event reconstruction are used to construct several BDTs to suppress backgrounds. The BDTs are trained for each jet multiplicity category to identify signal events that are generated either for $\kappa_{\mathrm{Hut}}(\mathrm{Hut})$ or $\kappa_{\mathrm{Hct}}(\mathrm{Hct})$ coupling against the sum of all background events. Separate trainings of the BDT for Hut and Hct are done. Distributions for some of the most discriminating BDT input variables, in the category with three jets, all of them b-tagged, can be seen in Figure 3. The final observable used to extract signal events is defined as the BDT score distribution. The resultant observed (expected) 95\% CL exclusion limits on top quark FCNC decay branching fractions are $\mathscr{B}(\mathrm{t} \rightarrow \mathrm{uH})<0.47 \%(0.34 \%)$ and $\mathscr{B}(\mathrm{t} \rightarrow \mathrm{cH})<0.47 \%(0.44 \%)$. Two-dimensional limits are also shown in Figure 4. We define a signal strength parameter $\mu=\sigma / \sigma_{\text {sig }}$, where $\sigma$ is the cross section excluded at $95 \% \mathrm{CL}$ and $\sigma_{\text {sig }}$ is the predicted cross section for signal. A maximum likelihood fit is performed for the signal strength, and is shown in Figure 4.

A CMS and ATLAS summary [4] of the current 95\% CL observed limits on the branching ratios of the top quark decays via FCNC to a quark and a neutral boson is shown in Figure 5. 

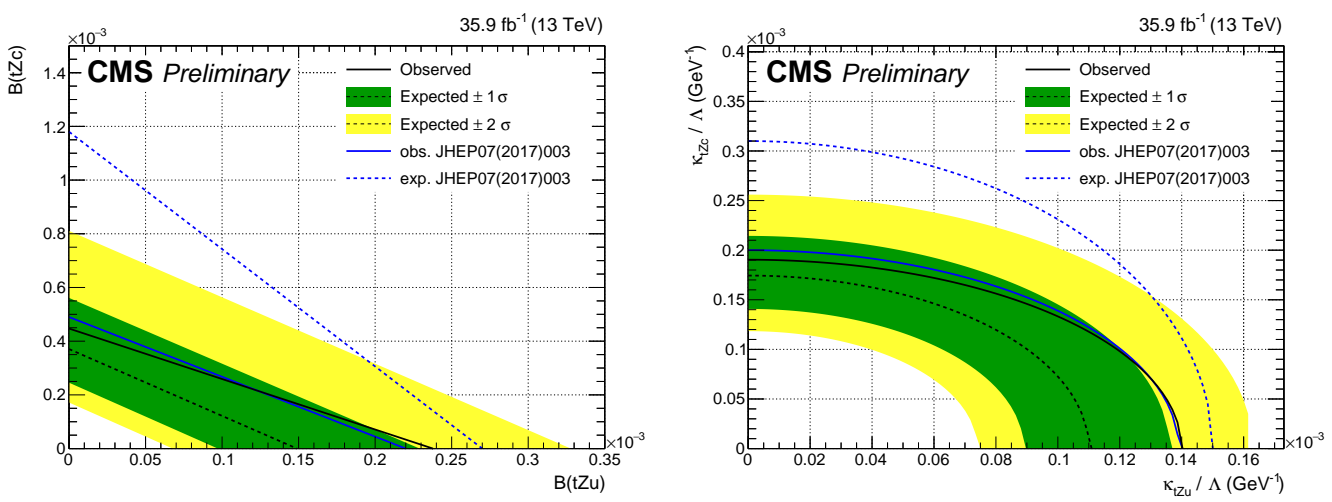

Figure 2: Exclusion regions at 95\% CL on the FCNC branching fractions (left) and couplings (right) in the 2D plane of both the $\mathrm{tZu}$ and $\mathrm{tZc}$ variables [1]. The CMS $8 \mathrm{TeV}$ observed (expected) limit is given with a blue line (dashed line).
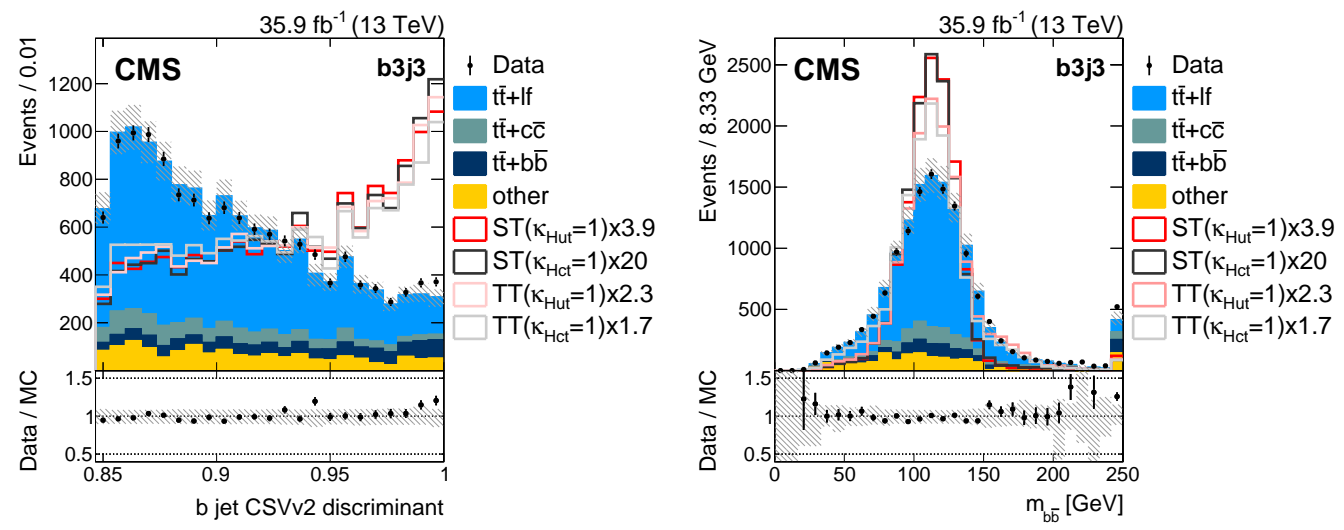

Figure 3: Comparison between data and simulation for some of the most discriminating BDT input variables in the category with three jets, all of them b-tagged [3]: CSV discriminant value for one of the reconstructed $\mathrm{b}$ jets assigned to Higgs boson decay (left), and reconstructed invariant mass of two $\mathrm{b}$ jets associated with the Higgs boson decay (right).

\section{Angular observables in $\mathrm{B}^{+} \rightarrow \mathrm{K}^{+} \mu^{+} \mu^{-}$}

In Reference [5] we present a study of the angular distribution of the FCNC decay $\mathrm{B}^{+} \rightarrow \mathrm{K}^{+} \mu^{+} \mu^{-}$ in pp collisions at the center-of-mass energy of $8 \mathrm{TeV}$. The analysis is based on data collected by the CMS detector at the LHC, corresponding to an integrated luminosity of $20.5 \mathrm{fb}^{-1}$. There are two model-independent parameters that describe the decay rate for the process: the forward/backward asymmetry $A_{F B}$ of the dimuon system and the contribution $F_{H}$ from the pseudo-scalar, scalar, and tensor amplitudes to the decay width. Because SM amplitudes may interefere with the contributions from non-SM particles inside loop diagrams, this decay channel can probe the presence of yet unobserved particles and processes. The decay rate for the process $\mathrm{B}^{+} \rightarrow \mathrm{K}^{+} \mu^{+} \mu^{-}$depends on $\cos \theta_{\ell}$, where $\theta_{\ell}$ is the angle between the direction of the $\mu^{-}$and the $\mathrm{K}^{+}$in the dilepton rest frame. The $\cos \theta_{\ell}$-dependence of the decay process can be parametrized in terms of the observables of interest $A_{F B}$ and $F_{H}$, which are extracted with an extended unbinned maximum-likelihood fit to 

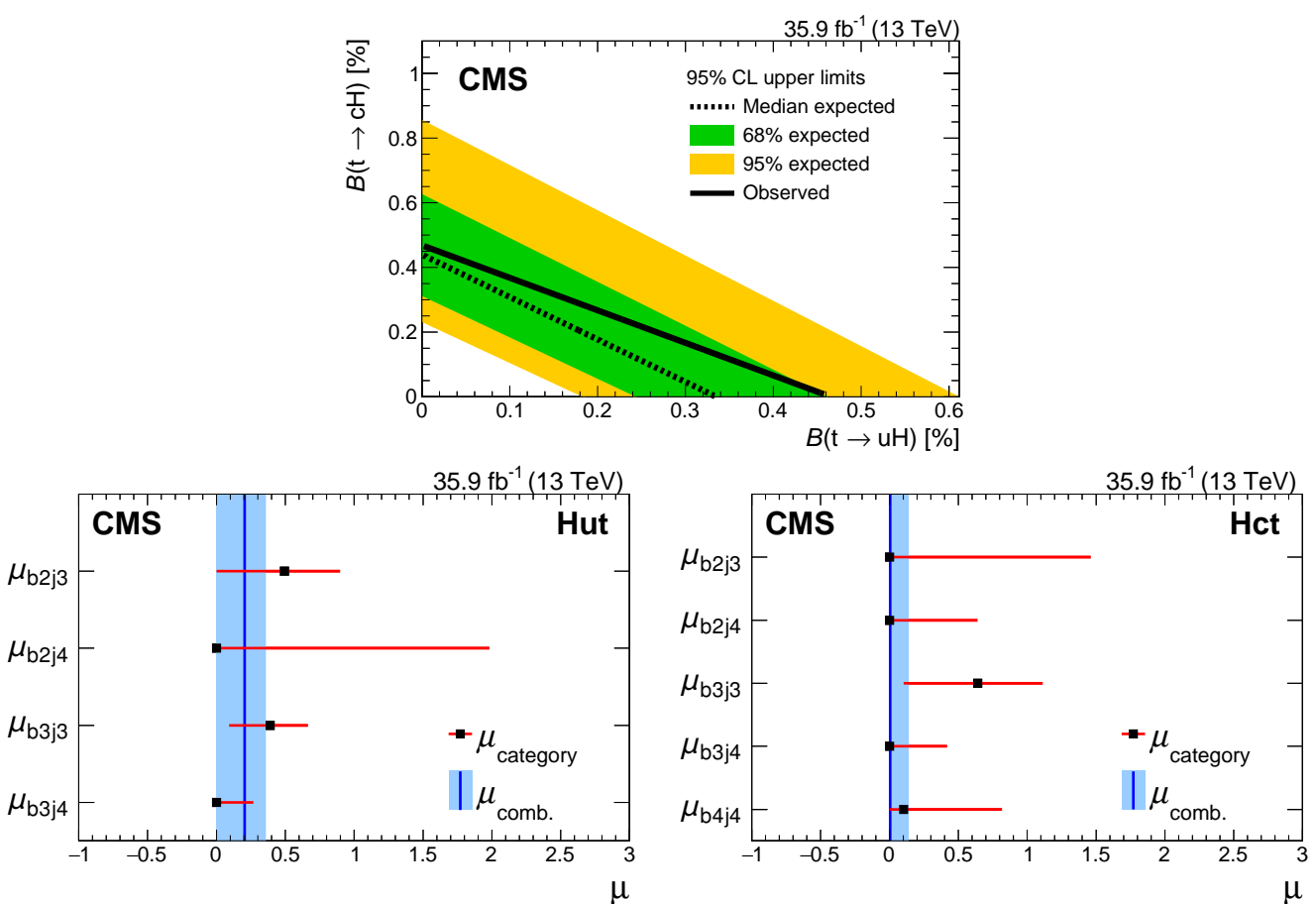

Figure 4: Upper limits on $\mathscr{B}(\mathrm{t} \rightarrow \mathrm{uH})$ and $\mathscr{B}(\mathrm{t} \rightarrow \mathrm{cH})$ at $95 \% \mathrm{CL}$ (top), and the best fit signal strength for Hut (bottom left) and Hct (bottom right), which is restricted to positive values in the fit [3].

the angular distributions of the selected B candidates in the data, in bins of $q^{2}$. Figure 6 shows the projections from the two-dimensional fits for the inclusive low- $q^{2}$ range $1-6 \mathrm{GeV}^{2}$. The measured values of $A_{F B}$ and $F_{H}$ for each $q^{2}$ bin are shown in Figure 7 (top distributions). The results are consistent with previous measurements and with various standard model predictions.

\section{Angular observables in $\mathrm{B}^{0} \rightarrow \mathrm{K}^{* 0} \mu^{+} \mu^{-}$}

In Reference [6] we study angular distributions of the decay $\mathrm{B}^{0} \rightarrow \mathrm{K}^{* 0} \mu^{+} \mu^{-}$using a sample of proton-proton at the center-of-mass energy of $8 \mathrm{TeV}$ collected with the CMS detector at the LHC, corresponding to an integrated luminosity of $20.5 \mathrm{fb}^{-1}$. An angular analysis is performed to determine the $P_{1}$ and $P_{5}^{\prime}$ parameters, where the $P_{5}^{\prime}$ parameter is of particular interest because of recent measurements that indicate a potential discrepancy with the standard model predictions [9, 10]. Based on a sample of 1397 signal events, the $P_{1}$ and $P_{5}^{\prime}$ parameters are determined as a function of the dimuon invariant mass squared, as can be seen in Figure 7 (bottom distributions). The measurements are in agreement with predictions based on the standard model. 


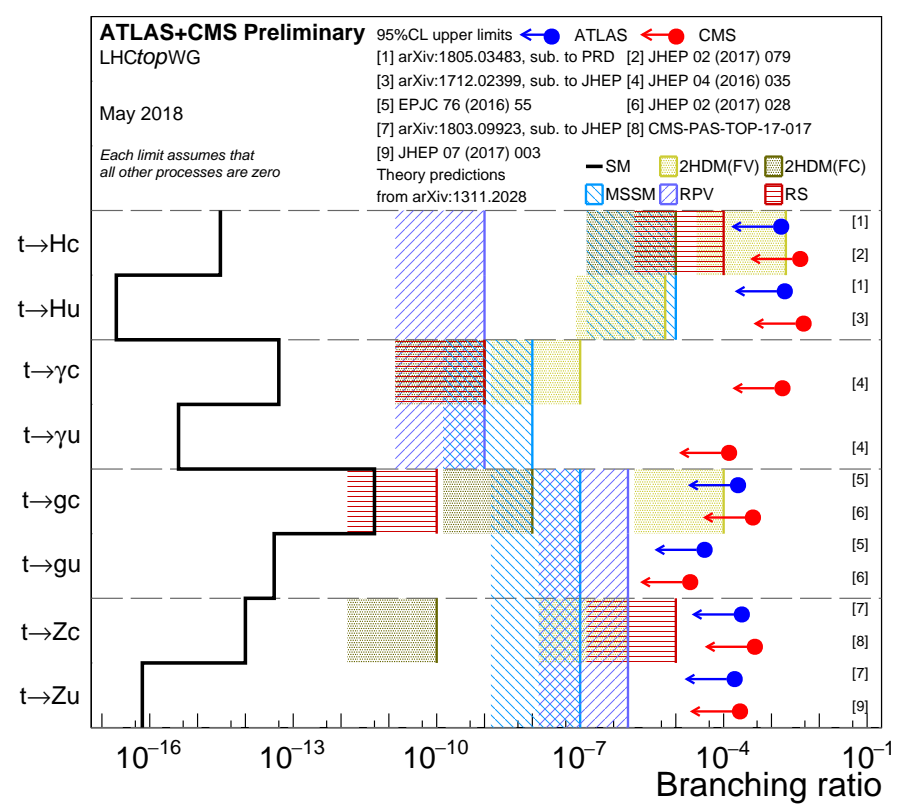

Figure 5: Summary of the current $95 \%$ confidence level observed limits on the branching ratios of the top quark decays via flavour changing neutral currents to a quark and a neutral boson by the ATLAS and CMS Collaborations compared to several new physics models [4].
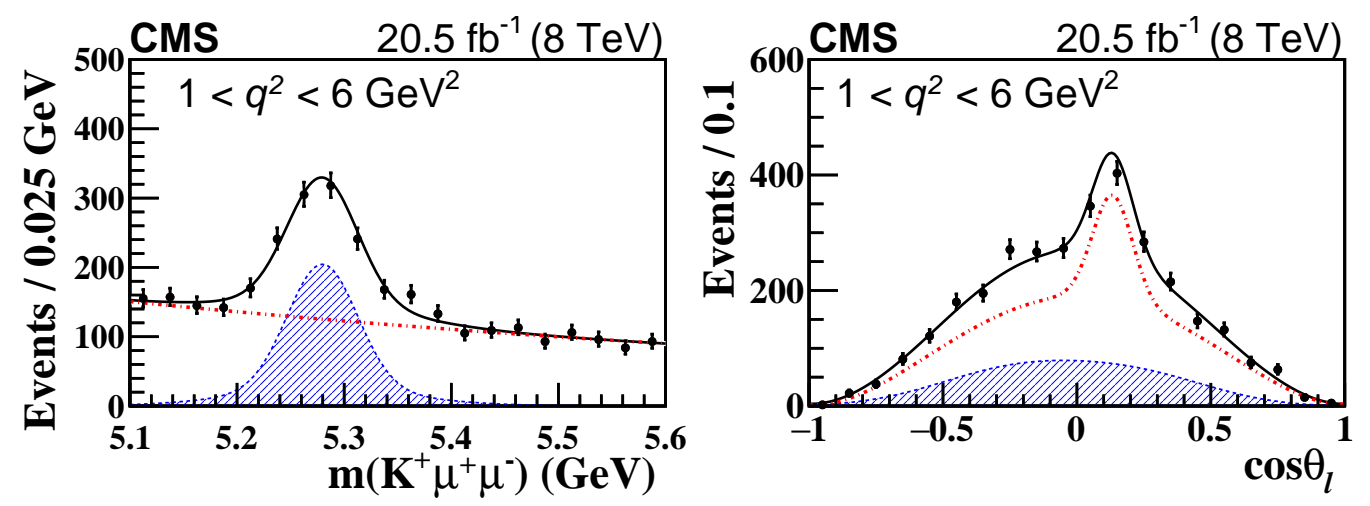

Figure 6: Projections of the $\mathrm{K}^{+} \mu^{+} \mu^{-}$invariant mass distribution (left) and the $\cos \theta_{\ell}$ distribution (right) from the two-dimensional fit of data, in the $1<\mathrm{q}^{2}<6 \mathrm{GeV}^{2}$ range [5]. The solid lines show the total fit, the shaded area the signal contribution, and the dash-dotted lines the background. 

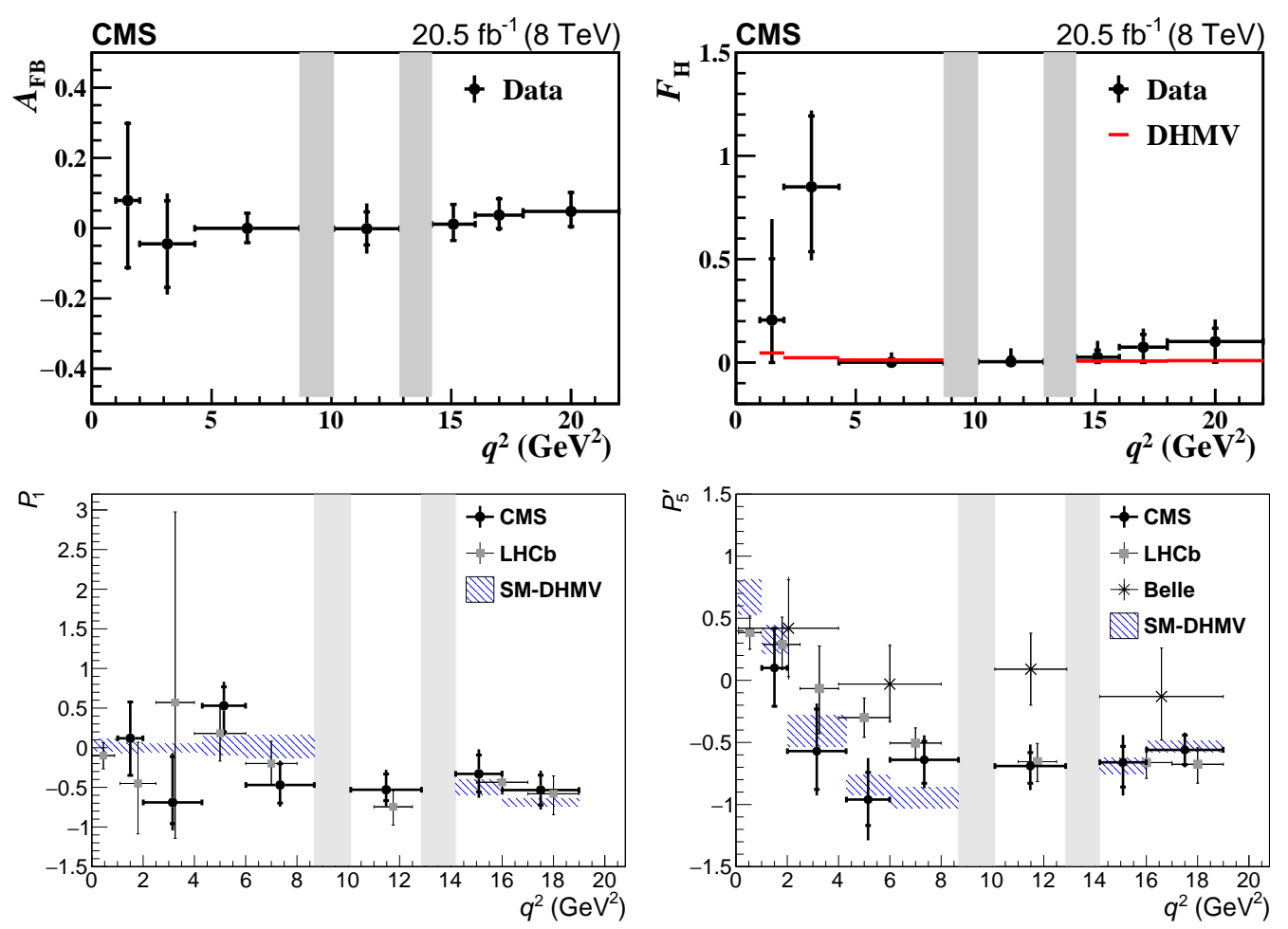

Figure 7: Measurements of the $A_{\mathrm{FB}}$ (top left) and $F_{\mathrm{H}}$ (top right) parameters versus $q^{2}$ for $\mathrm{B}^{+} \rightarrow \mathrm{K}^{+} \mu^{+} \mu^{-}$ decays [5]. Measurements of the $P_{1}$ (bottom left) and $P_{5}^{\prime}$ (bottom right) angular parameters versus $q^{2}$ for $\mathrm{B}^{0} \rightarrow \mathrm{K}^{* 0} \mu^{+} \mu^{-}$decays [6]. The CMS results are compared to SM DHMV theoretical predictions [7, 8] and, for the $P_{1}$ and $P_{5}^{\prime}$ parameters, they are also compared to results from the LHCb [9] and Belle [10] Collaborations. 


\section{References}

[1] CMS Collaboration, Search for flavour changing neutral currents in top quark production and decays with three-lepton final state using the data collected at $\sqrt{s}=13 \mathrm{TeV}$, https://cds.cern.ch/record/2292045, CMS-PAS-TOP-17-017.

[2] CMS Collaboration, The CMS experiment at the CERN LHC, JINST 3 S08004 (2008).

[3] CMS Collaboration, Search for the flavor-changing neutral current interaction of the top quark and the Higgs boson which decays into a pair of $b$ quarks at $\sqrt{s}=13 \mathrm{TeV}$, accepted for publication in JHEP https://cds.cern.ch/record/2296416, CERN-EP-2017-309 [a rXiv: 1712 . 02399v2].

[4] ATLAS and CMS Collaborations, https://twiki.cern.ch/twiki/pub/LHCPhysics/LHCTopWGSummaryPlots/fcnc_summarybsm_may18.pdf.

[5] CMS Collaboration, Angular analysis of the decay $\mathrm{B}^{+} \rightarrow \mathrm{K}^{+} \mu^{+} \mu^{-}$at $\sqrt{\mathrm{s}}=8 \mathrm{TeV}$, https://cds.cern.ch/record/2621370 CERN-EP-2018-125 [arXiv:1806.00636v2].

[6] CMS Collaboration, Measurement of angular parameters from the decay $\mathrm{B}^{0} \rightarrow \mathrm{K}^{* 0} \mu^{+} \mu^{-}$at $\sqrt{\mathrm{s}}=8 \mathrm{TeV}$, https://cds.cern.ch/record/2287571, CERN-EP-2017-240 [arXiv: $1710.02846 \mathrm{v} 2$ ].

[7] S. Descotes-Genon, L. Hofer, J. Matias, and J. Virto, On the impact of power corrections in the prediction of $B \rightarrow K^{*} \mu^{+} \mu^{-}$observables, JHEP 12 (2014) 125, [arXiv: 1407 . 852 6].

[8] S. Descotes-Genon, L. Hofer, J. Matias, and J. Virto, Global analysis of $b \rightarrow$ sll anomalies, JHEP 06 (2016) 092, [arXiv:1510.04239].

[9] LHCb Collaboration, Angular analysis of the $B^{0} \rightarrow K^{*} \mu^{+} \mu^{-}$decayusing $3 \mathrm{fb}^{-1}$ of integrated luminosity, JHEP 02 (2016) 104, [arXiv: 1512 : 044 42].

[10] Belle Collaboration, Lepton-flavor-dependent angular analysis of $B \rightarrow K^{*} \ell^{+} \ell^{-}$, Phys. Rev. Lett. 118 (2017) 111801, [arXiv:1612.05014]. 